\title{
PAIRS TRADING WITH OPPORTUNITY COST
}

\author{
CARL LINDBERG, ${ }^{*}$ The Second Swedish National Pension Fund
}

\begin{abstract}
Pairs trading is a trading strategy which is used very frequently in the financial industry. An investment opportunity arises when the spread between two assets, which historically have exhibited autoregressive behavior, deviates from its recent history. In this case, the investor takes a long position in the asset which is expected to outperform going forward and finances this by taking a short position in the other one. If the spread converges, the investor can close both positions to generate a profit. We model the spread between two assets as an Ornstein-Uhlenbeck process and assume a constant opportunity cost. We then study the optimal liquidation strategy for an investor who wants to optimize profit in excess of the opportunity cost. Including this cost is important from an applied perspective, as the performance of any investment is always evaluated relative to the performance of the opportunity set.
\end{abstract}

Keywords: Optimal stopping; pairs trading; opportunity cost; optimization; trading

2010 Mathematics Subject Classification: Primary 91G80; 60G40

\section{Introduction}

A pair trade is a portfolio consisting of two positions, a long position in one asset, partly or fully financed by a short position in another one. These two positions are thought of as being a single trade, so that the long and short legs are taken on and exited simultaneously. Hence, a pair trade can, in principle, eliminate the market risk and instead be exposed only to relative market movements. The standard assumption in pairs trading is that the spread between the legs of the pair trade is autoregressive. If the spread is observed to be significantly displaced relative to its perceived mean level, then the investor buys the asset in the pair which is expected to outperform going forward. This is financed by a short position in the other asset. If the spread converges, the pair trade can be liquidated to make a profit.

There appears to be limited academic literature on pairs trading given its very widespread applications in the financial industry. For a historical evaluation of pairs trading, see [4]. In [3], discrete time pair spreads are modeled as autoregressive processes of order 1 . The authors of [2] model the spread in continuous time as Ornstein-Uhlenbeck processes, and solve the problem of when it is optimal to liquidate a pair trade. An analogous problem is solved in [6], where the spread is modeled by an Ornstein-Uhlenbeck process with jumps. The books [1], [8], and [9] treat some applied aspects of pairs trading.

The present note assumes the same spread model as in [2], namely that the difference $X$ between two assets is

$$
\mathrm{d} X(t)=-\mu X(t) \mathrm{d} t+\sigma \mathrm{d} W(t),
$$

where $\mu, \sigma>0$. We extend the problem in [2] to include also an opportunity cost, which is a key concept in economics and finance. The opportunity cost is essentially the value of the

Received 31 August 2012; revision received 7 March 2013.

* Postal address: The Second Swedish National Pension Fund, Box 11155, Gothenburg, 404 24, Sweden. Email address: carl.lindberg@ap2.se 
best available alternative which was not chosen. Of course, this alternative changes over time. To include an opportunity cost is a very important extension from an applied perspective. The reason is that an investor will always view a trade relative to its opportunity set. The opportunity cost is assumed to have constant rate of growth.

The model risk associated to pairs trading is quite significant in the sense that the mean reverting behavior for a spread eventually always breaks down. Hence, we have to account for this in our model. The model error is, of course, hard to spot; is a large displacement in a spread a very good pair trade opportunity or a sign of a model failure? We adopt the stop-loss approach to handle model errors, as in [2] and [6]. This approach, which is industry standard, states that we believe in the assumed model up to a predetermined loss level $B<0$, at which we abandon the model and liquidate the position to accept the loss.

We set up the optimal stopping problem in Section 2. It is solved in Section 3, where we also verify that the optimal stopping barrier is monotonically decreasing in the rate of the opportunity cost.

\section{The optimal stopping problem}

We model by $X$ a portfolio consisting of a long position in stock $S_{1}$ and a short position in stock $S_{2}$, i.e.

$$
X(t)=\gamma_{1} S_{1}(t)-\gamma_{2} S_{2}(t)
$$

for $\gamma_{1}, \gamma_{2}>0$. We assume that $X$ is an Ornstein-Uhlenbeck process with dynamics

$$
\mathrm{d} X(t)=-\mu X(t) \mathrm{d} t+\sigma \mathrm{d} W(t),
$$

where $\mu, \sigma>0$, and $W$ is a Brownian motion. Note that there is no loss of generality to assume that $X$ has stationary mean 0 .

We define the value function as

$$
V(x)=\sup _{\tau \leq \tau_{B}} \mathbb{E}_{x}[X(\tau)-c \tau],
$$

where $c$ is the rate of the opportunity cost, and the supremum is taken over all stopping times that are smaller than

$$
\tau_{B}=\inf \left\{t: X_{t} \leq B\right\},
$$

the first hitting time of the stop-loss liquidation level $B<0$.

The opportunity cost is the sacrifice related to not investing in the second best of all available choices. As this alternative varies over time, it is natural to model it as a constant running cost. From an applied perspective, the inclusion of an opportunity cost in the model is a very important extension. This is because an investor will always benchmark a trade to its opportunity set.

Note that stopping at $B$ gives the pair trade an upper bound on the possible losses. Further, if we knew that the model (1) would hold in the future, there would be no reason to have a stop-loss. This is because we would then know that regardless of how dislocated the spread was, it would eventually revert back to generating a profit on the trade. However, in practice, the mean reversion will eventually break down, urging that we impose a stop-loss level.

\section{The solution}

We define the process $Y$ to have the dynamics

$$
\mathrm{d} Y(t)=\mathrm{d} X(t)-c \mathrm{~d} t=-(\mu X(t)+c) \mathrm{d} t+\sigma \mathrm{d} W(t) .
$$


It is immediately obvious that the drift of $Y$ is positive if $X(t)<-c / \mu$ and negative if $X(t)>-c / \mu$. Hence, if $B \geq-c / \mu$ then $Y\left(t \wedge \tau_{B}\right)$ is a supermartingale and $\tau=0$ is optimal. If $B<-c / \mu$, we expect from general optimal stopping theory, see [7], that the optimal stopping time is of the form $\tau^{*}=\tau_{B} \wedge \tau^{b}$, where

$$
\tau^{b}=\inf \{t \geq 0: X(t) \geq b\}
$$

for some $b>-c / \mu$ which is to be determined. Furthermore, the pair $(V, b)$ solves

$$
\begin{aligned}
\frac{\sigma^{2}}{2} V_{x x}-\mu x V_{x}=c & \text { if } x \in(B, b), \\
V(B) & =B, \\
V(b) & =b, \\
V^{\prime}(b) & =1 .
\end{aligned}
$$

The general solution to the ordinary differential equation $\frac{1}{2} \sigma^{2} V_{x x}-\mu x V_{x}=c$ is

$$
F(x)=\int_{0}^{x} \mathrm{e}^{\alpha y^{2} / 2}\left(\int_{0}^{y} \lambda \mathrm{e}^{-\alpha z^{2} / 2} \mathrm{~d} z+C_{1}\right) \mathrm{d} y+C_{2},
$$

where $\alpha:=2 \mu / \sigma^{2}, \lambda=2 c / \sigma^{2}$, and $C_{1}, C_{2}$ are constants. Inserting the general solution into the free-boundary problem (3), we obtain that the liquidation boundary must solve

$$
\int_{B}^{b} \mathrm{e}^{\alpha y^{2} / 2}\left(-\int_{y}^{b} \lambda \mathrm{e}^{-\alpha z^{2} / 2} \mathrm{~d} z+\mathrm{e}^{-\alpha b^{2} / 2}\right) \mathrm{d} y-(b-B)=0 .
$$

Lemma 1. Equation (4) admits a unique solution $b$ larger than $B$ if $-c / \mu>B$. Moreover, $b \in(-c / \mu,-B)$.

Proof. We define

$$
g(x)=\int_{B}^{x} \mathrm{e}^{\alpha y^{2} / 2}\left(-\int_{y}^{x} \lambda \mathrm{e}^{-\alpha z^{2} / 2} \mathrm{~d} z+\mathrm{e}^{-\alpha x^{2} / 2}\right) \mathrm{d} y-(x-B),
$$

and note that $b>B$ is a solution of (4) if and only if $g(b)=0$. We have $g(B)=0$, and

$$
g^{\prime}(x)=-(\lambda+\alpha x) \mathrm{e}^{-\alpha x^{2} / 2} \int_{B}^{x} \mathrm{e}^{\alpha y^{2} / 2} \mathrm{~d} y \geq 0
$$

if $x \in[B,-c / \mu]$ and $g^{\prime}(x)<0$ if $x>-c / \mu$. Moreover,

$$
g(-B)=\int_{B}^{-B} \mathrm{e}^{\alpha y^{2} / 2}\left(-\int_{y}^{-B} \lambda \mathrm{e}^{-\alpha z^{2} / 2} \mathrm{~d} z+\mathrm{e}^{-\alpha B^{2} / 2}\right) \mathrm{d} y+2 B<0
$$

since $\lambda>0$. It follows that $g$ has a unique zero $x=b \in(-c / \mu,-B)$.

Given the unique solution $b$ of (4), we can verify that the candidate solution $(\hat{V}, b)$ defined as

$$
\hat{V}(x)= \begin{cases}\int_{B}^{x} \mathrm{e}^{\alpha y^{2} / 2}\left(-\int_{y}^{b} \lambda \mathrm{e}^{-\alpha z^{2} / 2} \mathrm{~d} z+\mathrm{e}^{-\alpha b^{2} / 2}\right) \mathrm{d} y+B, & B \leq x<b, \\ x, & x \geq b\end{cases}
$$

is the unique solution to the free boundary problem (3). Note also that $\hat{V}(x) \geq x$ for all $x \geq B$, by the proof of Lemma 1 above. 
Theorem 1. The value function $V$ coincides with the function $\hat{V}$ given in (6). Moreover, $\tau^{*}=\tau_{B} \wedge \tau^{b}$ is an optimal stopping time in (2).

Proof. Consider the process $Z(t)=\hat{V}\left(X\left(t \wedge \tau_{B}\right)\right)$. By a generalized version of Itô's lemma,

$$
\begin{aligned}
Z(t)= & \hat{V}(x)+\int_{0}^{t \wedge \tau_{B}}\left(\frac{\sigma^{2}}{2} \hat{V}_{x x}(X(s))-\mu X(s) \hat{V}_{x}(X(s))\right) \mathbf{1}(X(s) \neq b) \mathrm{d} s \\
& +\int_{0}^{t \wedge \tau_{B}} \sigma \hat{V}_{x}(X(s)) \mathbf{1}(X(s) \neq b) \mathrm{d} W(s) \\
= & \hat{V}(x)+\int_{0}^{t \wedge \tau_{B}} c \mathbf{1}(X(s)<b) \mathrm{d} s-\mu \int_{0}^{t \wedge \tau_{B}} X(s) \mathbf{1}(X(s)>b) \mathrm{d} s \\
& +\int_{0}^{t \wedge \tau_{B}} \sigma \hat{V}_{x}(X(s)) \mathrm{d} W(s) .
\end{aligned}
$$

Let $\tau$ be a stopping time. The optional sampling theorem (see, e.g. Problem 3.16 and Theorem 3.22 of [5]) gives

$$
\begin{aligned}
& \mathbb{E}\left[X_{\tau \wedge \tau_{B}}-\int_{0}^{\tau \wedge \tau_{B}} c \mathrm{~d} s\right] \\
& \quad \leq \mathbb{E} \hat{V}\left(X\left(\tau \wedge \tau_{B}\right)\right)-\mathbb{E}\left[\int_{0}^{\tau \wedge \tau_{B}} c \mathrm{~d} s\right] \\
& \quad=\hat{V}(x)-\mathbb{E}\left[\int_{0}^{\tau \wedge \tau_{B}}(c+\mu X(s)) \mathbf{1}(X(s)>b) \mathrm{d} s+\int_{0}^{\tau \wedge \tau_{B}} \sigma \hat{V}_{x}(X(s)) \mathrm{d} W(s)\right] \\
& \quad=\hat{V}(x)+\mathbb{E}\left[M_{B}(\tau)\right]
\end{aligned}
$$

where

$$
M_{B}(t)=\mathbb{E}\left[-\int_{0}^{t \wedge \tau_{B}}(c+\mu X(s)) \mathbf{1}(X(s)>b) \mathrm{d} s+\int_{0}^{t \wedge \tau_{B}} \sigma \hat{V}_{x}(X(s)) \mathrm{d} W(s)\right] .
$$

The Itô integral is a martingale since the integrand is bounded. Therefore, the process $M_{B}$ is a supermartingale since $b>-c / \mu$, so $\hat{V}(x)+\mathbb{E}\left[M_{B}(\tau)\right] \leq \hat{V}(x)$. Hence,

$$
V(x) \leq \hat{V}(x)
$$

since $\tau$ is arbitrary.

Now, note that $Z\left(t \wedge \tau^{b}\right)$ is a bounded martingale, and that $Z\left(\tau^{b}\right)=X\left(\tau_{B} \wedge \tau^{b}\right)$. It follows that the inequalities in (7) reduce to equalities for $\tau=\tau^{*}$. This completes the proof.

We prove now that the liquidation region is monotone in the model parameter $B$ and $c$.

Theorem 2. The optimal stopping boundary $b$ is decreasing as a function of the parameters $B$ and $c$.

Proof. We recall that $\lambda=2 c / \sigma^{2}$. Define

$$
g(b, B, \lambda):=\int_{B}^{b} \mathrm{e}^{\alpha y^{2} / 2}\left(-\int_{y}^{b} \lambda \mathrm{e}^{-\alpha z^{2} / 2} \mathrm{~d} z+\mathrm{e}^{-\alpha b^{2} / 2}\right) \mathrm{d} y-(b-B),
$$


and denote by $g_{i}^{\prime}$ the differential with respect to the $i$ th argument. The proof consists of applying the implicit function theorem to the relation $g(b, B, \lambda)=0$. From (5), we have

$$
g_{1}^{\prime}(b, B, \lambda)<0
$$

since $b>-c / \mu$ by Lemma 1 . Further calculations give

$$
g_{2}^{\prime}(b, B, \lambda)=\int_{B}^{b}(\lambda+\alpha x) \mathrm{e}^{-\alpha x^{2} / 2} \mathrm{~d} x .
$$

But, from (5) and the fundamental theorem of calculus, we have

$$
\int_{B}^{b}(\lambda+\alpha x) \mathrm{e}^{-\alpha x^{2} / 2} \int_{B}^{x} \mathrm{e}^{\alpha y^{2} / 2} \mathrm{~d} y \mathrm{~d} x=0 .
$$

We note that $h(x):=1 / \int_{B}^{x} \mathrm{e}^{\alpha y^{2} / 2} \mathrm{~d} y$ is decreasing in $x$ on $(B, b)$, and define the function $\tilde{h}=h+C$, for some constant $C$, such that $\tilde{h}(\beta)=0$. We set $f:=(\lambda+\alpha x) \mathrm{e}^{-\alpha x^{2} / 2} \int_{B}^{x} \mathrm{e}^{\alpha y^{2} / 2} \mathrm{~d} y$, which gives

$$
\begin{aligned}
g_{2}^{\prime}(b, B, \lambda) & =\int_{B}^{b} f(x) h(x) \mathrm{d} x \\
& =\int_{B}^{b} f(x) \tilde{h}(x) \mathrm{d} x-C \int_{B}^{b} f(x) \mathrm{d} x \\
& =\int_{B}^{-c / \mu} f(x) \tilde{h}(x) \mathrm{d} x+\int_{-c / \mu}^{b} f(x) \tilde{h}(x) \mathrm{d} x \\
& <0 .
\end{aligned}
$$

It is immediate that $g_{3}^{\prime}<0$. The implicit function theorem gives the results.

There is no monotonicity of the liquidation barrier $b$ with respect to the parameters $\mu$ and $\sigma$.

\section{Acknowledgement}

The author wishes to thank an anonymous referee for insightful comments which helped to improve the paper.

\section{References}

[1] Ehrman, D. S. (2006). The Handbook of Pairs Trading: Strategies Using Equities, Options, and Futures. John Wiley, Hoboken, NJ.

[2] Ekström, E., LindBerg, C. AND Tysk, J. (2011). Optimal liquidation of a pairs trade. In Advanced Mathematical Methods for Finance, Springer, Heidelberg, pp. 247-255.

[3] Elliott, R. J., van der Hoek, J. and Malcolm, W. P. (2005). Pairs trading. Quant. Finance 5, 271-276.

[4] Gatev, E., Goetzmann, W. N. and Rouwenhorst, K. G. (2006). Pairs trading: performance of a relative-value arbitrage rule. Rev. Financial Studies 19, 797-827.

[5] Karatzas, I. and Shreve, S. E. (1991). Brownian Motion and Stochastic Calculus. Springer, New York.

[6] Larsson, S., Lindberg, C. AND WarfHeimer, M. (2013). Optimal closing of a pair trade with a model containing jumps. Appl. Math. 58, 249-268.

[7] Peskir, G. And Shiryaev, A. (2006). Optimal Stopping and Free-Boundary Problems. Birkhäuser, Basel.

[8] Vidyamurthy, G. (2004). Pairs Trading: Quantitative Methods and Analysis. John Wiley, Hoboken, NJ.

[9] Whistler, M. (2004). Trading Pairs: Capturing Profits and Hedging Risk with Statistical Arbitrage Strategies. John Wiley, Hoboken, NJ. 